\title{
Constructive rescue of TFIIH instability by an alternative isoform of XPD derived from a mutated XPD allele in mild but not severe XP-D/CS
}

\author{
Katsuyoshi Horibata ${ }^{1,6}$, Sayaka Kono ${ }^{1}$, Chie Ishigami ${ }^{1}$, Xue Zhang ${ }^{1}$, Madoka Aizawa ${ }^{2}$, Yuko Kako ${ }^{2}$, \\ Takuma Ishii ${ }^{3,4}$, Rika Kosaki ${ }^{5}$, Masafumi Saijo ${ }^{1}$ and Kiyoji Tanaka ${ }^{1}$
}

Mutations in XPD cause xeroderma pigmentosum (XP), XP and Cockayne syndrome (CS) crossover syndrome (XP/CS), trichothiodystrophy and cerebro-oculo-facio-skeletal syndrome (COFS). COFS represents the most severe end of the CS spectrum. This study reports two Japanese patients, COFS-05-135 and COFS-Chiba1, who died at ages of $<1$ year and exhibited typical COFS manifestations caused by XPD mutations p.[1619del];[R666W] and p.[G47R];[1619del], respectively. Two other cases of severe XP-D/CS (XP group D/CS), XP1JI (p.[G47R];[0]) and XPCS1PV (p.[R666W];[0]), died at ages $<2$ years. On the other hand, two cases of mild XP-D/CS, XP1NE (p.[G47R];[L461V;V716_R730del]) and XPCS118LV (p.[L461V;V716_R730del];[R666W]), lived beyond 37 years of age. p.1619Del and p.[L461V;V716_R730del] are functionally null; therefore, despite the differences in clinical manifestations, the functional protein in all of these patients was either p.G47R or p.R666W. To resolve the discrepancies in these XPD genotype-phenotype relationships, the p.[L461V;V716_R730del] allele was analyzed and we found that p.[L461V; A717G] was expressed from the same allele as p.[L461V;V716_R730del] by authentic splicing. Additionally, p.[L461V;A717G] could partially rescue the loss of XPD function, resulting in the milder manifestations observed in XP1NE and XPCS118LV. Journal of Human Genetics (2015) 60, 259-265; doi:10.1038/jhg.2015.18; published online 26 February 2015

\section{INTRODUCTION}

Cerebro-oculo-facio-skeletal syndrome (COFS) is an autosomal recessive disorder characterized by severe mental retardation, cataracts, optic atrophy, progressive joint contractures and postnatal growth deficiency. ${ }^{1} \mathrm{XPD}$ is one of the genes mutated in COFS; such mutations confer a DNA repair deficiency. ${ }^{2}$ In addition to COFS, mutations in $X P D$ cause xeroderma pigmentosum $(\mathrm{XP})^{3,4}$ and the XP and Cockayne syndrome (CS) crossover syndrome (XP/CS). ${ }^{5} \mathrm{CS}$ is characterized by abnormalities in physical and neurological development, with dysmyelination but no predisposition to skin cancer. ${ }^{6}$ Both COFS and CS are dysmorphology syndromes, and COFS shares many clinical and cellular similarities with $\mathrm{CS}^{7}$ Therefore, COFS is considered to represent the most severe end of the CS spectrum; ${ }^{8}$ the phenotypic differences between COFS and CS are caused by variations in the mutations that occur in the responsible genes. The XPD protein is involved in nucleotide excision repair (NER), ${ }^{9}$ a versatile DNA repair system that removes a wide range of DNA lesions, including those induced by UV damage. Because XPD is one of the components of basal transcription factor TFIIH and has a dual role in transcription and NER, ${ }^{10-12}$ the various manifestations observed in COFS, XP and
XP/CS patients are attributed to complex abnormalities in both DNA repair and transcription. ${ }^{13-17}$ In this study, we describe two Japanese COFS patients with compound heterozygous mutations in XPD, including the novel XPD p.I619del mutation in both patients. We further analyzed the genotype-phenotype relationships between these patients and other XP-D/CS (XP group D/CS) patients who exhibited mild or severe XP-D/CS clinical features, and found that a third mutated XPD protein, p.[L461V;A717G], was expressed because of authentic splicing in patients who exhibited mild XP-D/CS clinical features. Additionally, we showed that the protein encoded by the $\mathrm{p}$. [L461V;A717G] allele could form TFIIH and partially rescue the UV hypersensitivity of an XPD-deficient cell line. These findings suggest that p.[L461V;A717G] can restore some aspects of XPD function, resulting in milder XP-D/CS symptoms.

\section{MATERIALS AND METHODS}

Ethical approvals from The Life Sciences Committee at Osaka University and Chiba Children's Hospital were obtained for all studies. The research was conducted according to the Principles of the Declaration of Helsinki. Informed consent for the studies was obtained from both patients' relatives.

${ }^{1}$ Human Cell Biology Group, Graduate School of Frontier Biosciences, Osaka University, Suita, Osaka, Japan; ²Division of Neonatology, Chiba Children's Hospital, Midori-ku, Chiba, Japan; ${ }^{3}$ Division of Clinical Genetics, Chiba Children's Hospital, Midori-ku, Chiba, Japan; ${ }^{4}$ Department of Pediatrics, Kawaguchi Kogyo General Hospital, Kawaguchi, Saitama, Japan and ${ }^{5}$ Division of Medical Genetics, National Center for Child Health and Development, Setagaya-ku, Tokyo, Japan

${ }^{6}$ Current address: Division of Genetics and Mutagenesis, National Institute of Health Sciences, Setagaya-ku, Tokyo, Japan.

Correspondence: Dr K Horibata or Professor K Tanaka, Division of Genetics and Mutagenesis, National Institute of Health Sciences, 1-18-1 Kamiyoga, Setagaya-ku, Tokyo 1588501, Japan. 


\section{UV survival assay}

Cells were inoculated into 10-cm dishes at a density of 1000-2000 cells per dish. After $6 \mathrm{~h}$, cells were washed with phosphate-buffered saline and UV irradiated. The cells were then incubated for 2-3 weeks. The colonies that formed were fixed with $10 \%$ formalin, stained with $0.1 \%$ crystal violet and counted under a binocular microscope.

\section{Sequencing analysis of XPD cDNA and genomic DNA}

Total RNA was prepared from fibroblast cell lines using the RNeasy Mini Kit (Qiagen, Tokyo, Japan). XPD cDNA was amplified using the Qiagen OneStep RT-PCR Kit (Qiagen) with the following primers: sense, 5'-GTC GACCCCGCTGCACAGTC-3'; antisense, 5'-CAGGAGTCACCAGGAAC CGTTTATG-3'. Genomic DNA was prepared from fibroblast cell lines using the QIAamp DNA Mini Kit (Qiagen). Genomic DNA fragments containing $X P D$ mutations were amplified by PCR using the following primers: p.G47R sense, 5'-AAGACCACTCAACAAGGAGGAGGC-3'; p.G47R antisense, 5'-TT CCCAAACACCCCCGAAAG-3'; p.I619del sense, 5'-GCTCTCTGGAACA CCTGCCCG-3'; p.I619del antisense, 5'-GAAATGAACGGGAAACAGCCTG G-3'; p.R666W sense, 5'-CTACACACAGAGCCGCATTCTCA-3'; p.R666W antisense $5^{\prime}$-GTCAGGTTGAGGTTGGCATCTG-3'.

The DNA sequences of the reverse transcription-PCR (RT-PCR) and PCR products were analyzed using the Big Dye Terminator Cycle Sequencing Kit (Life Technologies Japan, Tokyo, Japan) on an ABI PRISM 3130 Genetic Analyzer (Life Technologies Japan).

\section{Unscheduled DNA synthesis after UV irradiation}

Unscheduled DNA synthesis after UV-irradiation (UV-UDS) tests were performed as described previously ${ }^{18}$ with some modifications. Polycarbonate beads with diameters of 0.7 or $2 \mu \mathrm{m}$ were added to each type of cells at $50 \%$ confluence in $10-\mathrm{cm}$ dishes. Three days after incubation, cells were trypsinized and washed five times with phosphate-buffered saline. Cells carrying large beads and small beads were mixed at 1:1 ratio, and then seeded on poly-L-lysine-coated coverslips in a 6-well culture plate (Corning Japan, Tokyo, Japan). UV-C light was irradiated at a dose of $20 \mathrm{~J} \mathrm{~m}^{-2}$ after overnight incubation. Cells were then labeled for $3 \mathrm{~h}$ in Dulbecco's modified Eagle's medium containing $50 \mu \mathrm{Ci} \mathrm{ml}^{-1}\left[{ }^{3} \mathrm{H}\right]$ thymidine, and then fixed with methanol-acetic acid (3:1). Coverslips were mounted on glass slides, dipped in Kodak NTB-3 emulsion and exposed for $96 \mathrm{~h}$ at $4{ }^{\circ} \mathrm{C}$. The slides were then imaged using developer and medical X-ray film fixer solution (Fujifilm, Tokyo, Japan) and stained with $2 \%$ Giemsa. Autoradiographic grains above the nuclei were counted under the microscope; S-phase cells were excluded. UV-UDS efficiency was calculated as the average number of grains in cells carrying large and small beads.

\section{RT-PCR/restriction fragment length polymorphism analysis}

The XPD cDNA fragment containing the c.2150C $>\mathrm{G}$ mutation (found in XP1NE) was amplified from total RNA purified from fibroblasts using the Qiagen OneStep RT-PCR Kit (Qiagen) and the following primers: sense, 5'-CCATCAGGGGCAAGACGGAC-3'; antisense, 5'-GTGGAAGGGCTGTGC CATCTG-3'. The amplified DNA fragments were digested with the restriction enzyme MscI (Takara Bio Inc., Otsu, Japan), and then subjected to electrophoresis on a $2 \%$ agarose gel.

\section{Immunoprecipitation of XPD protein}

The N-terminally HA- and C-terminally FLAG-tagged cDNA of XPD p.WT was cloned into the expression vector pCAGGS. p.[L461V;A717G] and p.[L461V;V716_R730del] cDNAs were prepared from XP1NE cells by RT-PCR and subcloning. Other mutant XPD cDNAs were prepared using QuikChange Site-Directed Mutagenesis Kit (Agilent Technologies, Tokyo, Japan), and then recloned into pCAGGS. XP6BESV cells stably expressing N-terminally HA- and C-terminally FLAG-tagged cDNA of p.WT, p.G47R, p. L461V, p.[L461V;A717G], p.[L461V;V716_R730del], p.I619del, p.R666W, p. A717G or p.V716_R730del were established. The protein complexes were immunoprecipitated from whole-cell extracts using anti-FLAG M2-conjugated agarose. Bound proteins were eluted with the FLAG peptide.
Sodium dodecyl sulfate-polyacrylamide gel electrophoresis and immunoblotting

Sodium dodecyl sulfate-polyacrylamide gel electrophoresis and immunoblotting were performed as described previously. Whole-cell extracts were separated by sodium dodecyl sulfate-polyacrylamide gel electrophoresis and transferred to PVDF membranes in blotting buffer ( $25 \mathrm{~mm}$ Tris- $\mathrm{HCl}$ ( $\mathrm{pH}$ 8.3), $0.2 \mathrm{M}$ glycine). After blocking, the blot was incubated for $1 \mathrm{~h}$ with specific antibodies, followed by the ECL Plus Western Blotting Detection System (GE Healthcare UK Ltd., Buckinghamshire, UK). Anti-XPB (S-19), anti-p62 (Q-19) and anti-XPG (8H7) antibodies were purchased from Santa Cruz Biotechnology (Dallas, TX, USA). Anti-p44 (TF2-1H5) and anti-HA (3F10) antibodies were purchased from Euromedex (Souffelweyersheim, France) and Roche Diagnostics K. K. (Tokyo, Japan), respectively.

\section{RESULTS}

\section{Clinical report of COFS-05-135}

Here we report a 10-month-old girl with COFS. A Japanese couple, both healthy and unrelated, was referred to us during their third pregnancy because of a past history of two abnormal pregnancies. The first pregnancy ended in early spontaneous abortion, and the second pregnancy resulted in a boy with COFS who died at 5 months. During the third pregnancy, fetal ultrasound at 33 weeks showed severe intrauterine growth retardation, and subsequent amniocentesis revealed a chromosomal complement of 46,XX. The girl was delivered vaginally at 39 weeks of gestation. The patient had a birth weight of $1850 \mathrm{~g}$, a length of $42 \mathrm{~cm}$ and a head circumference of $29.3 \mathrm{~cm}$. Apgar scores were 7 at $1 \mathrm{~min}$ and 9 at $5 \mathrm{~min}$. Physical examination revealed microcephaly, microphthalmia, deep-set eyes with prominent nasal root, large ear pinnae, micrognathia, camptodactyly, moderate flexion contractures in the elbows and knees and kyphosis. Ophthalmologic examination revealed hypoplasia of the macula lutea and microcornea, and audiological evaluation revealed profound bilateral hearing impairment. Based on the phenotype and family history, the girl was diagnosed as having COFS. The patient exhibited profound growth and developmental delay, with no head control at 6 months. At 7 months, she had episodes of seizures compatible with a diagnosis of West syndrome. At 9 months, she developed a cutaneous erythematous lesion, later diagnosed by biopsy as Langerhans cell histiocytosis. She died of respiratory insufficiency at the age of 12 months.

\section{Clinical report of COFS-Chibal}

This male infant was born after 37 weeks of gestation as the fourth offspring of non-consanguineous normal Japanese parents with no history of miscarriage or stillbirth. The patient exhibited a prenatal growth deficit (birth weight, $1820 \mathrm{~g}$ ) as well as classic COFS facial features, including microcephaly, bilateral microphthalmia with deepset eyes, prominent nasal root, prominent large ear pinnae and micrognathia. In addition, the patient had bilateral simian creases and widely set nipples. He manifested failure to thrive, progressive multiple joint contractures, no developmental progress, infantile spasm and obvious photosensitivity. He died before 1 year of age from pneumonia, at which time his weight was $3000 \mathrm{~g}$. His eldest brother, who died at 5 months of age with leukocytosis and proteinuria, had similar features: intrauterine growth retardation, microcephaly, bilateral microphthalmia, failure to thrive, progressive multiple joint contractures, no developmental progress and obvious photosensitivity. In both siblings, chromosomes of peripheral lymphocytes revealed a normal 46,XY male karyotype. Two other male siblings were normal. 
a

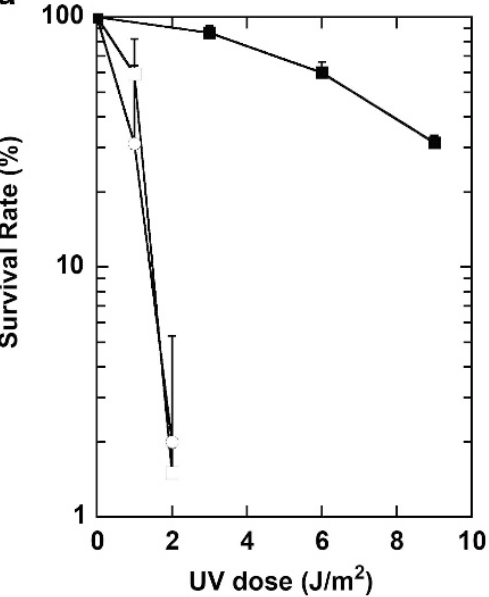

d

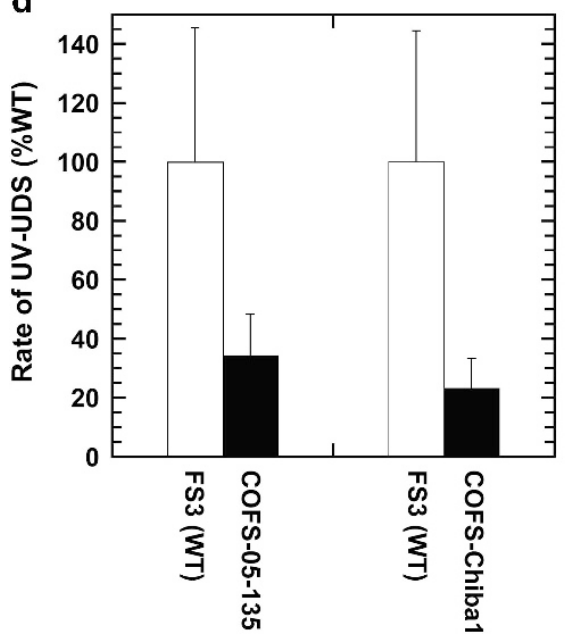

b

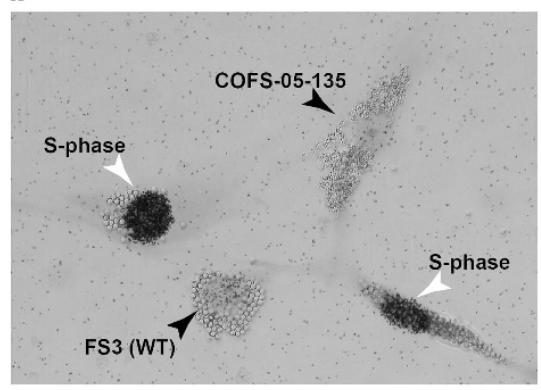

e

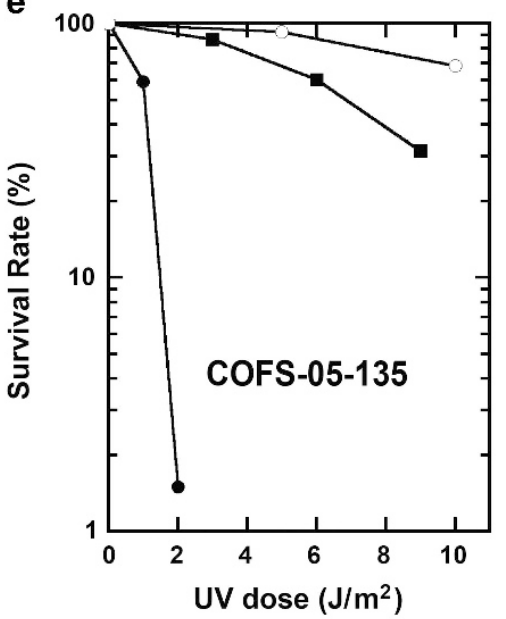

c

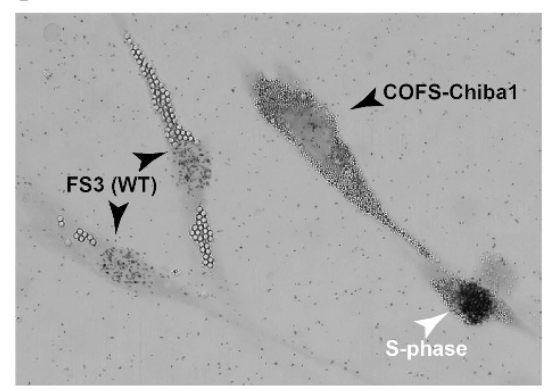

f

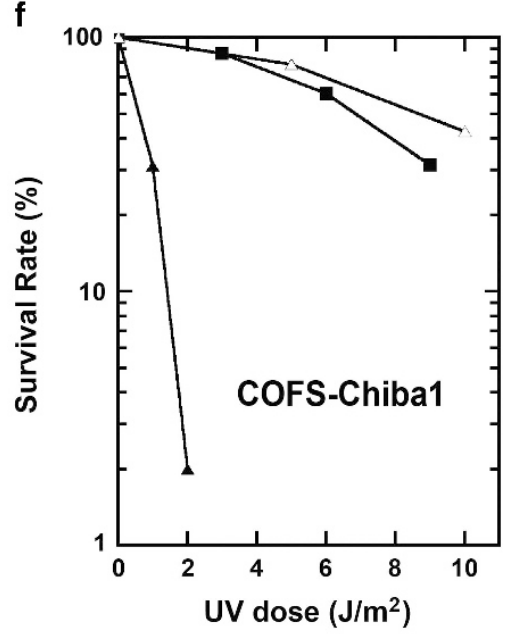

Figure 1 Cellular characteristics of COFS-05-135 and COFS-Chiba1 cells. (a) After UV treatment, survival of WI38VA13 cells (closed squares), COFS-05-135 cells (open squares) and COFS-Chibal cells (open circles). Both patients' cell lines exhibited UV hypersensitivity. (b and c) UV-UDS test of COFS-05-135 cells (b) and COFS-Chibal cells (c). S-phase cells are indicated by open arrowheads. (d) Quantitative measurement of UV-UDS tests in (b and c). Grains from $n=55$ cells were counted under the microscope. (e) After UV treatment, survival of p.WT/COFS-05-135 cells (that is, COFS-05-135 cells expressing XPD p.WT) (open circles), COFS-05-135 cells (closed circles) and WI38VA13 cells (closed squares). (f) After UV treatment, survival of p.WT/COFSChibal cells (open triangles), COFS-Chibal cells (closed triangles) and WI38VA13 cells (closed squares). Error bars represent the s.d. from the mean of three independent experiments.

Cellular characterization of COFS cells

UV survival assay revealed that primary fibroblast cell lines derived from both COFS-05-135 and COFS-Chiba1 were hypersensitive to UV irradiation (Figure 1a), indicating that both patients were defective in nucleotide excision repair (NER) of UV damage. Two subpathways exist in NER: ${ }^{9}$ transcription-coupled NER, which preferentially removes DNA lesions from the transcribed strand of active genes that block ongoing transcription, and global genome NER, which removes lesions throughout the genome. To determine which pathway was defective in these patients, we analyzed the level of UV-UDS, a hallmark of global genome NER. Cells from both COFS patients exhibited reduced levels of UV-UDS relative to control cells (Figures $1 \mathrm{~b}-\mathrm{d}$ ), indicating that both patients were defective in global genome NER. Because mutations in the XPB, XPD and XPG genes have been associated with $\mathrm{XP} / \mathrm{CS}$, we analyzed these three genes and detected XPD mutations in both patients: c. [1855_1857del];[1996C $>$ T] in COFS-05-135, resulting in XPD p.[I619del];[R666W]; and c. [139G $>$ A];[1855_1857del] in COFS-Chibal, resulting in XPD p.
[Gly47Arg];[Ile619del] (see Supplementary Information). Stable expression of wild-type XPD (p.WT) in COFS-05-135 and COFSChibal cells (p.WT/COFS-05-135 and p.WT/COFS-Chiba1) complemented the UV hypersensitivity of COFS-05-135 and COFS-Chiba1 cells (Figures 1e and f). Therefore, we concluded that COFS in both these patients was caused by mutations in the XPD gene.

\section{Genotype-phenotype relationships in XP-D/CS patients}

Compound heterozygous XP-D/CS patients with p.G47R or p.R666W are summarized in Table 1 . In XP1JI and XPCS1PV, the only expressed allele was p.G47R and p.R666W, respectively. ${ }^{19,20}$ p.[L461V;V716_R730del], found in XP1NE and XPCS118LV, is functionally null in NER and TFIIH. ${ }^{3,21,22}$ Because p.I619del could not form the TFIIH complex, as in the case of p.[L461V; V716_R730del] (Figure 3c, lanes 5 and 6), we considered p.I619del to be functionally null. These observations mean that the only functional XPD protein in these patients was p.G47R or p.R666W; however, XP1NE and XPCS118LV exhibited considerably milder 
Table 1 Summary of genotype-phenotype relationships of the XPD alleles used in this study

\begin{tabular}{llllll}
\hline Patients & Diagnosis & Age at death & XPD mutation in allele 1 & XPD mutation in allele 2 & Reference \\
\hline XP1NE & XP-D/CS (mild) & 43 years & p.G47R & p.L461V;V716_R730del p.L461V;A717G & 3,9,21, this paper \\
XP1JI & XP-D/CS (severe) & 24 months & p.G47R & p.0 & 19,20 \\
COFSS-Chiba1 & COFS & 5 months & p.G47R & p.I619del & This paper \\
XPCS118LV & XP-D/CS (mild) & $>37$ years & p.L461V;V716_R730del p.L461V;A717G & p.R666W & 20,23, this paper \\
XPCS1PV & XP-D/CS (severe) & 15 months & p.0 & p.R666W & 20 \\
COFSS-05-135 & COFS & 12 months & p.I619del & p.R666W & This paper \\
\hline
\end{tabular}

Abbreviations: COFS, cerebro-oculo-facio-skeletal syndrome; XP-D/CS, xeroderma pigmentosum group D and Cockayne syndrome crossover syndrome.

a

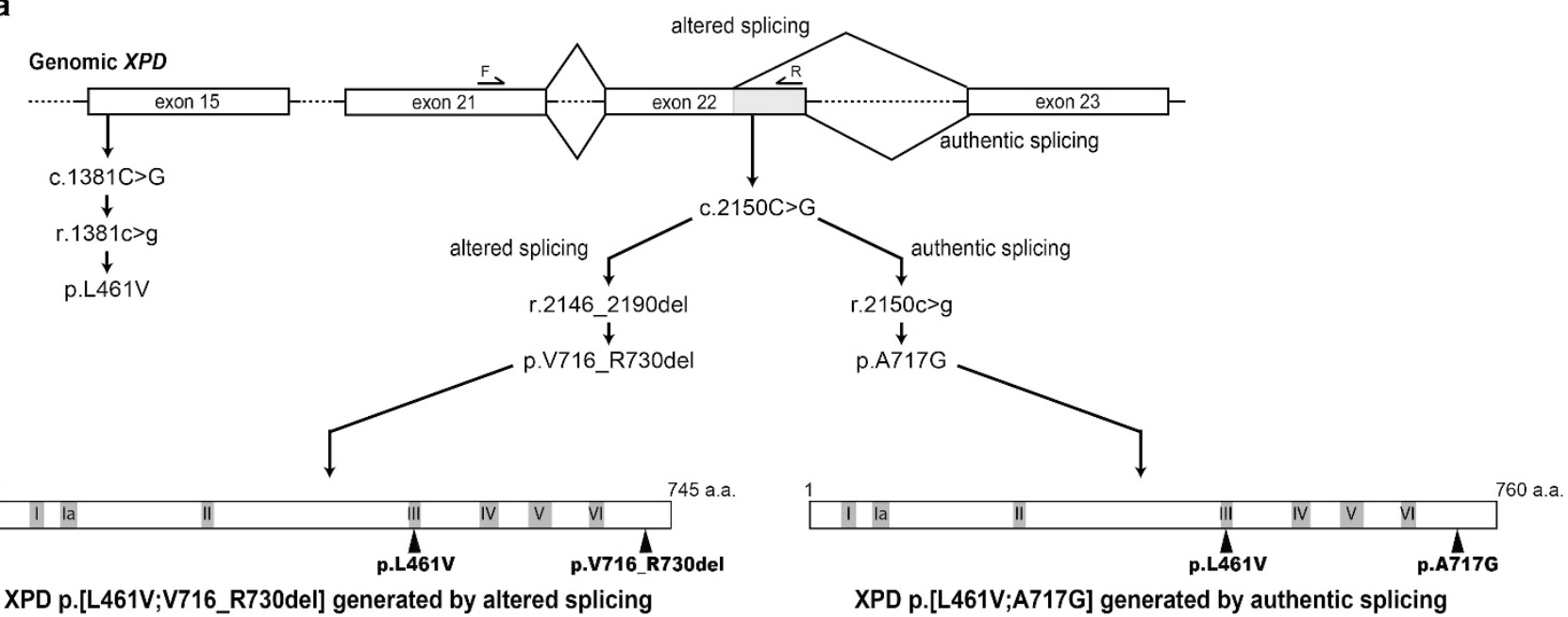

b
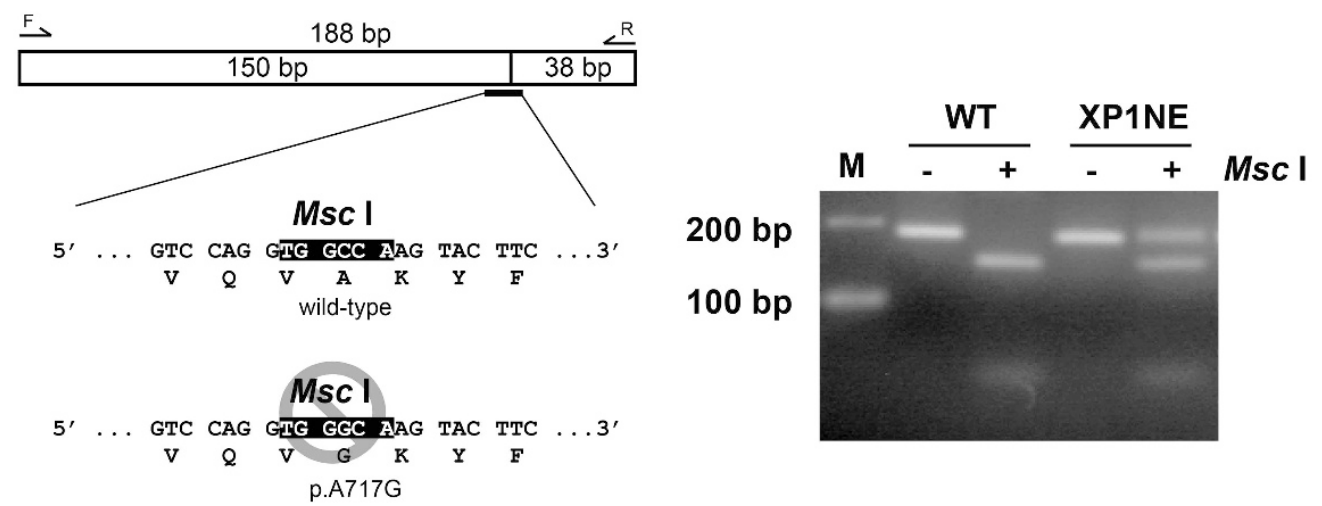

Figure 2 Analyses of XPD alleles of severe and mild XP-D/CS patients. (a) In the XPD allele of mild XP-D/CS patients, a c.1381C > G mutation was located on exon 15, and a c.2150C > G mutation was located on exon 22, resulting in a novel splice donor. This new splice donor gives rise to altered splicing, resulting in the expression of $p .\left[L 461 V ; V 716 \_R 730 d e l\right]$. Additionally, XPD mRNA r.2150c $>g$, the alternative isoform arising from the $c .1381 C>G$ mutation, was generated by authentic splicing, resulting in the expression of p.[L461V;A717G]. (b) RT-PCR/restriction fragment length polymorphism (RFLP) analysis of the $\mathrm{c} .2150 \mathrm{C}>\mathrm{G}$ mutation. The mutation site in the XPD cDNA of XP1NE corresponds to the recognition site of the restriction enzyme Mscl. XPD cDNA containing the mutation site was amplified by RT-PCR using primers located on exons 21 and 22, shown by arrowheads on the upper side of (a), and then digested with Mscl. The RT-PCR products derived from WI38VA13 cells were cut with Mscl, generating 150- and 38-bp fragments, whereas the XPD cDNA containing the c.2150C $>$ G heterozygous mutation from XP1NE was not cut; this is visible as the 188-bp fragment that appears in addition to the digested 150- and 38-bp fragments.

symptoms and survived more than 10 times longer than XP1JI, XPCS1PV, COFS-05-135 and COFS-Chiba1 (Table 1). To resolve this discrepancy in the genotype-phenotype relationships, we analyzed the allele encoding p.[L461V;V716_R730del]. In addition to the c.1381C $>$ G mutation resulting in p.L461V, the c.2150C $>$ G mutation on exon 22 generated a splice donor. The novel splice site gave rise to altered splicing, resulting in r.[1381c >g;2146_2190del], which is translated into p.[L461V;V716_R730del] (Figure 2a). ${ }^{20,21}$ Based on the presence of this c.2150C $>\mathrm{G}$ mutation, we predicted that authentic splicing could generate a normal-length mutated XPD mRNA, r.[1381c $>$ g;2150c $>$ g], which would be translated into p.[L461V; A717G] (Figure 2a). Because the c.2150C $>$ G mutation site corresponds to the recognition site of the restriction enzyme $M s c \mathrm{I}$, we were able to perform RT-PCR/restriction fragment length polymorphism 
a

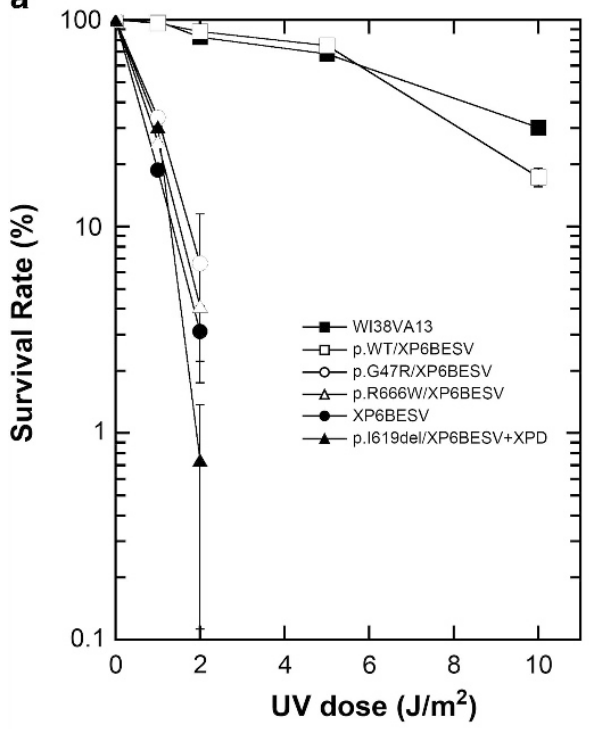

b

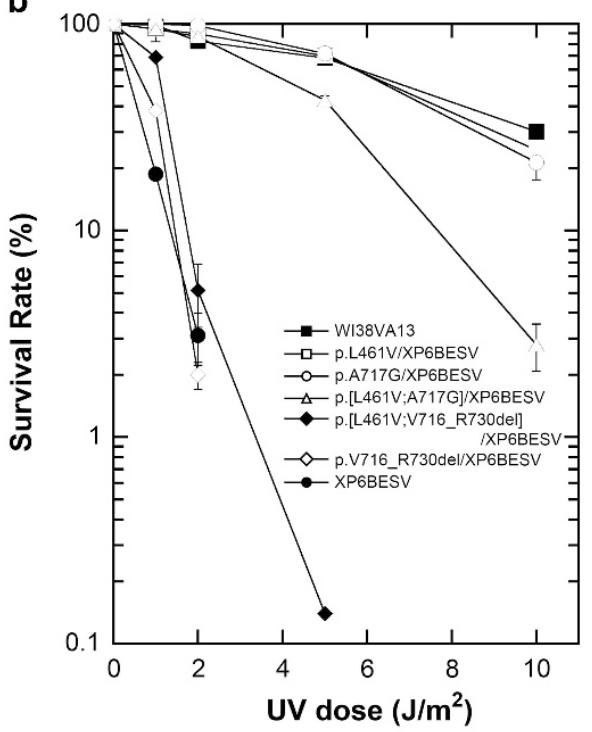

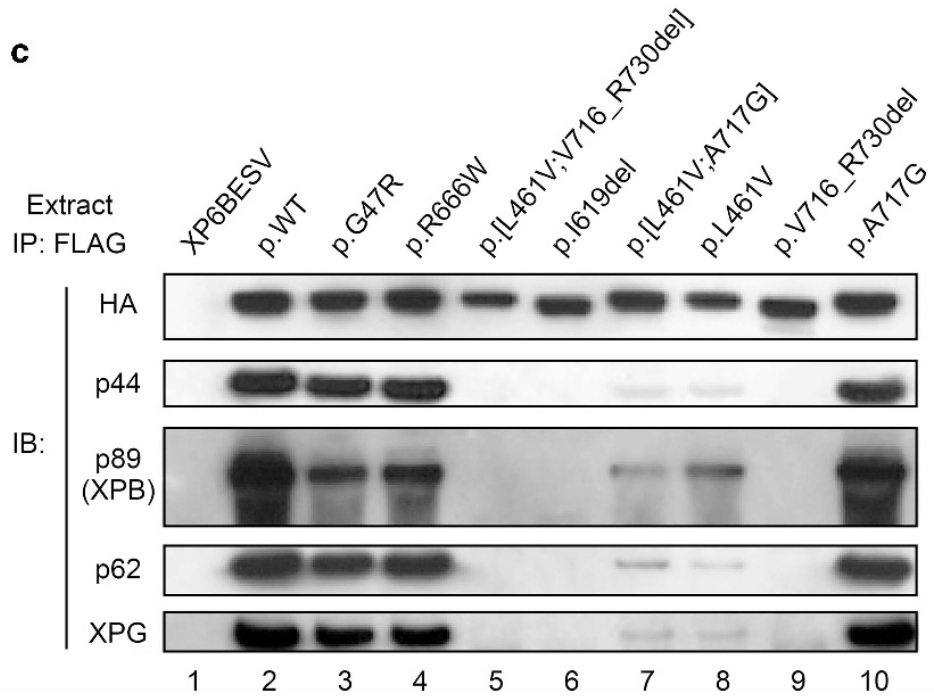

Figure 3 Functional analyses of mutated XPDs. (a) After UV treatment, survival of WI38VA13 (closed squares), p.WT/XP6BESV (open squares), p.G47R/ XP6BESV (open circles), p.R666W/XP6BESV (open triangles), XP6BESV (closed circles) and p.I619del/XP6BESV (closed triangles). (b) After UV treatment survival of p.L461V/XP6BESV (open squares), p.A717G/XP6BESV (open circles), p.[L461V;A717G]/XP6BESV (open triangles), p.[L461V;V716_R730del]/ XP6BESV (closed diamonds) and p.V716_R730del/XP6BESV (open diamonds). (c) Immunoprecipitation of XPD protein. N-terminally HA- and C-terminally FLAG-tagged XPD proteins were stably expressed in each XP6BESV-derived cell line shown in (a) and (b). The protein complexes were immunoprecipitated from whole-cell extracts with anti-FLAG M2-conjugated agarose. Elutants were subjected to sodium dodecyl sulfate-polyacrylamide gel electrophoresis (SDSPAGE) and immunoblotting, using the antibodies indicated on the left side of (c). Error bars represent the s.d. from the mean of three independent experiments.

analysis. As shown in Figure 2b, the RT-PCR products derived from WT cells were fully cut by MscI, generating 150- and 38-bp fragments, whereas the RT-PCR products derived from XP1NE primary fibroblasts generated both cut and uncut fragments. Additionally, fulllength c. [1381C $>$ G;2150C $>$ G] cDNA was subcloned from XP1NE cells and used in subsequent experiments. These results indicated that p.[L461V;A717G] was expressed from the p.[L461V;V716_R730del]encoding allele as a result of authentic splicing.

Functional analysis of mutated XPDs

To examine the NER activity of each mutant XPD protein found in these six patients, we established stable transfectants expressing $\mathrm{N}$-terminal HA- and C-terminal FLAG-tagged XPD proteins, including
WT, in the XPD-deficient cell line XP6BESV (p.WT/XP6BESV, p.G47R/XP6BESV, p.L461V/XP6BESV, p.[L461V;V716_R730del]/ XP6BESV, p.[L461V;A717G]/XP6BESV, p.I619del/XP6BESV, p.R666W/ XP6BESV, p.V716_R730del/XP6BESV and p.A717G/XP6BESV). We then subjected these transfectants to UV survival assays. p.WT/XP6BESV, p.L461V/XP6BESV and p.A717G/XP6BESV cells exhibited the same level of UV resistance as WI38VA13 (WT) (Figures 3a and b). By contrast, p.G47R/XP6BESV, p.[L461V; V716_R730del]/XP6BESV, p.I619del/XP6BESV, p.R666W/XP6BESV and p.V716_R730del/XP6BESV exhibited the same UV hypersensitivity as XP6BESV cells. Interestingly, p.[L461V;A717G]/XP6BESV exhibited UV hypersensitivity intermediate between that of WI38VA13 and XP6BESV (Figures 3a and b). These results indicated that p.G47R, 
p.[L461V;V716_R730del], p.I619del, p.R666W and p.V716_R730del were completely defective in NER of UV-induced DNA damage. Additionally, although p.L461V and p.A717G possessed full NER activity (comparable to p.WT), the combined mutant p.[L461V; A717G] possessed only partial NER activity.

\section{TFIIH-forming activity of mutated XPDs}

The results of the UV survival assays shown in Figures $3 a$ and $b$ prompted us to investigate the ability of the XPD variants to form the TFIIH complex. To this end, we subjected whole-cell extracts from XP6BESV cells expressing various XPD proteins to immunoprecipitation with anti-FLAG antibodies. p.G47R, p.R666W and p.A717G could bind components of TFIIH, including XPG, as efficiently as p.WT (Figure 3c, lanes 2-4 and 10), whereas p.I619del, p.V716_R730del and p.[L461V;V716_R730del] could not (Figure 3c, lanes 5, 6 and 9). Additionally, p.L461V and p.[L461V;A717G] coimmunoprecipitated with components of TFIIH, including XPG, but only weakly (Figure 3c, lanes 7 and 8). These results indicated that p.G47R, p.R666W and p.A717G could form TFIIH complex normally, whereas p.I619del, p.V716_R730del and p.[L461V;V716_R730del] could not. Additionally, p.L461V and p.[L461V;A717G] formed the TFIIH complex to some extent, but they could not do so as efficiently as the WT protein.

\section{DISCUSSION}

We describe here two Japanese patients who exhibited COFS symptoms caused by mutations in the XPD gene. XPD gene mutations give rise to XP and XP/CS in addition to COFS. On the basis of XPD genotype-phenotype relationships, different clinical symptoms were strongly associated with various mutations in the $X P D$ gene.

COFS-Chiba1 and COFS-05-135, described in this report, and XP-D/CS patients XP1JI and XPCS1PV, described previously, ${ }^{19,20,23}$ had very severe symptoms and died at ages of 5 months to 2 years (Table 1). COFS represents the most severe end of the CS spectrum; ${ }^{8}$ therefore, we refer to these cases here as severe-XP-D/CS. p.I619Del, found in the two COFS patients described here, did not bind to the core complex of TFIIH (Figure 3c, lane 6) and could not rescue the NER defect of XP1BESV cell lines (Figure 3b). From these findings, we conclude that p.I619del is functionally null, and that only p.G47R or p.R666W was expressed as functional XPD in these COFS patients; single expression of p.G47R and p.R666W was the cause of severe XP-D/CS in XP1JI and XPCS1PV, respectively. ${ }^{19,20}$ Both p.G47R and p.R666W could bind and form TFIIH complex components, including XPG (Figure 3c, lanes 3 and 4), but neither protein could rescue the NER defect of XP6BESV cell lines (Figure 3a), indicating that both p.G47R and p.R666W completely lack NER activity. Previous biochemical examination revealed that p.G47R is defective in ATPase, helicase, DNA repair synthesis and dual-incision activities. ${ }^{24}$ There is no detailed biochemical information regarding human p.R666W, but structural and biochemical analyses have been performed on the Sulfolobus acidocaldarius XPD homolog. ${ }^{25}$ The Sulfolobus acidocaldarius XPD mutants p.G34R and p.R514W, which, respectively, correspond to human XPD p.G47R and p.R666W, are defective in ATPase and helicase activities. These biochemical results indicate that p.G47R and p.R666W have lost the ability to engage in basal transcription; nevertheless, both mutants can still form TFIIH complex (Figure 3c, lanes 3 and 4). Based on these observations, we conclude that sole expression of p.G47R or p.R666W caused the severe manifestations found in these patients.
On the other hand, XP1NE and XPCS118LV exhibited typical XP features along with neurological disease, and both patients survived beyond 30 years of age: XP1NE and XPCS118LV died at 43 and 37 years, respectively. ${ }^{19,20,23}$ Here we refer to these milder symptoms as mild XP-D/CS. A previous report indicated that p.[G47R];[L461V; V716_R730del] and p.[L461V;V716_R730del];[R666W] are the causes of XP1NE and XPCS118LV, respectively. ${ }^{20,21}$ However, we observed that authentic splicing resulted in the p.A717G amino-acid change, and that consequently, p.[L461V;A717G] was expressed from the p.[L461V;V716_R730del]-encoding allele (Figure 2). Therefore, it is clear that three kinds of mutated XPD protein are expressed in XP1NE (p.[G47R];[L461V;A717G,V716_R730del]) and XPCS118LV (p.[L461V;A717G,V716_R730del];[R666W]), as shown in Table 1. The p.[L461V;V716_R730del] mutation cannot rescue lethality in a rad15 mutant of Schizosaccharomyces pombe. ${ }^{21}$ Furthermore, biochemical analysis revealed that the single p.V716_R730del mutant protein lacks the ability to bind to p44 (ref. 22) and has a defect in basal transcription. ${ }^{24}$ Additionally, p.L461V has NER activity comparable to that of p.WT, although the p.L461V mutant forms a loose conformation of TFIIH (Figures $3 \mathrm{~b}$ and $\mathrm{c}$ ). These observations indicate that p.[L461V;V716_R730del] is functionally null because of the p.V716_R730del mutation. p.A717G formed TFIIH as competently as p.WT (Figure $3 c$, lane 10) and rescued the UV hypersensitivity of XP6BESV as well as p.WT (Figure 3b), indicating that p.A717G might possess full XPD function. In the case of compound-mutated XPD, that is, p.[L461V;A717G], the ability to bind core TFIIH was reduced, as in the case of p.L461V (Figure 3c, lane 10), suggesting that the p.L461V mutation affected the binding ability of p.A717G. Interestingly, p.[L461V;A717G] had partial NER activity (Figure 3b). These observations suggest that the low TFIIH-binding capacity of p. [L461V; A717G] might affect basal transcription and cause the mild XP-D/CS clinical features, although the basal transcription ability of p.[L461V; A717G] remains unknown.

The p.[L461V;V716_R730del]-encoding allele has been considered to be functionally null. ${ }^{20,21,23}$ However, we conclude that coexpression of p.[L461V;A717G] because of authentic splicing in the mild XP-D/CS patients partially rescues the functional defect of p.G47R or p.R666W, resulting in a more than 10 -fold increase in lifespan in mild XP-D/CS patients relative to patients with severe XP-D/CS.

\section{CONFLICT OF INTEREST}

The authors declare no conflict of interest.

\section{ACKNOWLEDGEMENTS}

This work was supported by a Grant-in-Aid for Scientific Research from the Ministry of Education, Culture, Sports, Science, and Technology (MEXT) of Japan.

1 Pena, S. D. \& Shokeir, M. H. Autosomal recessive cerebro-oculo-facio-skeletal (COFS) syndrome. Clin. Genet. 5, 285-293 (1974).

2 Graham, J. M. Jr, Anyane-Yeboa, K., Raams, A., Appeldoorn, E., Kleijer, W. J. \& Garritsen, V. H. et al. Cerebro-oculo-facio-skeletal syndrome with a nucleotide excisionrepair defect and a mutated XPD gene, with prenatal diagnosis in a triplet pregnancy. Am. J. Hum. Genet. 69, 291-300 (2001).

3 Frederick, G. D., Amirkhan, R. H., Schultz, R. A. \& Friedberg, E. C. Structural and mutational analysis of the xeroderma pigmentosum group D (XPD) gene. Hum. Mol. Genet. 3, 1783-1788 (1994).

4 Takayama, K., Salazar, E. P., Lehmann, A., Stefanini, M., Thompson, L. H. \& Weber, C. A. Defects in the DNA repair and transcription gene ERCC2 in the cancerprone disorder xeroderma pigmentosum group D. Cancer Res. 55, 5656-5663 (1995).

5 Broughton, B. C., Thompson, A. F., Harcourt, S. A., Vermeulen, W., Hoeijmakers, J. H. \& Botta, E. et al. Molecular and cellular analysis of the DNA repair defect in a patient in xeroderma pigmentosum complementation group $D$ who has the clinical features of 
xeroderma pigmentosum and Cockayne syndrome. Am. J. Hum. Genet. 56, 167-174 (1995).

6 Nance, M. A. \& Berry, S. A. Cockayne syndrome: review of 140 cases. Am. J. Med. Genet. 42, 68-84 (1992).

7 Graham, J. M. Jr., Hennekam, R., Dobyns, W. B., Roeder, E. \& Busch, D. MICRO syndrome: an entity distinct from COFS syndrome. Am. J. Med. Genet. Part A 128A, 235-245 (2004)

8 Laugel, V., Dalloz, C., Tobias, E. S., Tolmie, J. L., Martin-Coignard, D. \& Drouin-Garraud, V. et al. Cerebro-oculo-facio-skeletal syndrome: three additional cases with CSB mutations, new diagnostic criteria and an approach to investigation. J. Med. Genet. 45, 564-571 (2008).

9 Hanawalt, P. C. \& Spivak, G. Transcription-coupled DNA repair: two decades of progress and surprises. Nature reviews. Nat. Rev. Mol. Cell Biol. 9, 958-970 (2008).

10 Drapkin, R., Reardon, J. T., Ansari, A., Huang, J. C., Zawel, L. \& Ahn, K. et al. Dual role of TFIIH in DNA excision repair and in transcription by RNA polymerase II. Nature 368, 769-772 (1994).

11 Schaeffer, L., Moncollin, V., Roy, R., Staub, A., Mezzina, M. \& Sarasin, A. et al. The ERCC2/DNA repair protein is associated with the class II BTF2/TFIIH transcription factor. EMBO J. 13, 2388-2392 (1994).

12 Egly, J. M. \& Coin, F. A history of TFIIH: two decades of molecular biology on a pivotal transcription/repair factor. DNA Repair (Amst) 10, 714-721 (2011).

13 Cleaver, J. E., Lam, E. T. \& Revet, I. Disorders of nucleotide excision repair: the genetic and molecular basis of heterogeneity. Nat. Rev. Genet. 10, 756-768 (2009).

14 Hashimoto, S. \& Egly, J. M. Trichothiodystrophy view from the molecular basis of DNA repair/transcription factor TFIIH. Hum. Mol. Genet. 18, R224-R230 (2009).

15 Stefanini, M., Botta, E., Lanzafame, M. \& Orioli, D. Trichothiodystrophy: from basic mechanisms to clinical implications. DNA Repair (Amst) 9, 2-10 (2010).

16 Fuss, J. O. \& Tainer, J. A. XPB and XPD helicases in TFIIH orchestrate DNA duplex opening and damage verification to coordinate repair with transcription and cell cycle via CAK kinase. DNA Repair (Amst) 10, 697-713 (2011).
17 DiGiovanna, J. J. \& Kraemer, K. H. Shining a light on xeroderma pigmentosum. J. Invest. Dermatol. 132, 785-796 (2012).

18 Kobayashi, T., Kuraoka, I., Saijo, M., Nakatsu, Y., Tanaka, A. \& Someda, Y. et al. Mutations in the XPD gene leading to xeroderma pigmentosum symptoms. Hum. Mut. 9, 322-331 (1997).

19 Fujimoto, M., Leech, S. N., Theron, T., Mori, M., Fawcett, H. \& Botta, E. et al. Two new XPD patients compound heterozygous for the same mutation demonstrate diverse clinical features. J. Invest. Dermatol. 125, 86-92 (2005).

20 Theron, T., Fousteri, M. I., Volker, M., Harries, L. W., Botta, E. \& Stefanini, M. et al. Transcription-associated breaks in xeroderma pigmentosum group $D$ cells from patients with combined features of xeroderma pigmentosum and Cockayne syndrome. Mol. Cell. Biol. 25, 8368-8378 (2005).

21 Taylor, E. M., Broughton, B. C., Botta, E., Stefanini, M., Sarasin, A. \& Jaspers, N. G. et al. Xeroderma pigmentosum and trichothiodystrophy are associated with different mutations in the XPD (ERCC2) repair/transcription gene. Proc. Natl Acad. Sci. USA 94, 8658-8663 (1997).

22 Coin, F., Marinoni, J. C., Rodolfo, C., Fribourg, S., Pedrini, A. M. \& Egly, J. M. Mutations in the XPD helicase gene result in XP and TTD phenotypes, preventing interaction between XPD and the p44 subunit of TFIIH. Nat. Genet. 20, 184-188 (1998)

23 Andressoo, J. O., Jans, J., de Wit, J., Coin, F., Hoogstraten, D. \& van de Ven, M. et al. Rescue of progeria in trichothiodystrophy by homozygous lethal Xpd alleles. PLoS Biol. 4, e322 (2006).

24 Dubaele, S., Proietti De Santis, L., Bienstock, R. J., Keriel, A., Stefanini, M. \& Van Houten, B. et al. Basal transcription defect discriminates between xeroderma pigmentosum and trichothiodystrophy in XPD patients. Mol. Cell 11, 1635-1646 (2003).

25 Fan, L., Fuss, J. O., Cheng, Q. J., Arvai, A. S., Hammel, M. \& Roberts, V. A. et al. XPD helicase structures and activities: insights into the cancer and aging phenotypes from XPD mutations. Cell 133, 789-800 (2008).

Supplementary Information accompanies the paper on Journal of Human Genetics website (http://www.nature.com/jhg) 